\title{
Effect of Atorvastatin on Pharmacology of Sitagliptin in Streptozotocin- Nicotinamide Induced Type-II Diabetes in Rats
}

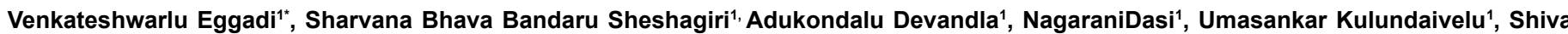 \\ Kumar Revoori ${ }^{2}$ and Sahith Reddy Kesireddy ${ }^{1}$
}

${ }^{1}$ Vaagdevi College of Pharmacy, Hanamkonda, Warangal, Telangana, India.

${ }^{2}$ Singhania University, Pacheri, Jhunjhun, Rajastan,India.

\begin{abstract}
Prolonged type 2 diabetes mellitus (Type2DM) may lead to high risk of cardiovascular disease (CVD) requiring a global therapeutic approach. Statin therapy has proven its efficacy in reducing CVD events in Type2 DM patients. Dipeptidyl peptidase-4 inhibitors (gliptins), which are increasingly used to target hyperglycemia. In the present study type 2 DM in rats by i.p. Administration of Streptozotocin (STZ); $(60 \mathrm{mg} / \mathrm{kg})$-Nicotinamide $(120 \mathrm{mg} / \mathrm{kg})$. Diabetes induced rats were divided into groups and treated with Sitagliptin alone and in combination with atorvastatin for 7 days. Blood samples were collected by retro orbital puncture. Mean glucose concentration was measured by GODPOD method using commercial glucose kits and sitagliptin in plasma was estimated by RP-HPLC method using methanol: water (60:40 v/v, containing $10 \mathrm{mM}$ Tris and $10 \mathrm{mM}$ Triethylamine) was adjusted to $\mathrm{pH} 9.0$ using $1 \mathrm{~mol} / \mathrm{L}$ hydrochloric acid. The blood glucose lowering activity of sitagliptin was increased by the presence of atorvastatin in diabetic rats. The pharmacokinetic (PK) and pharmacodynamic (PD) parameters of sitagliptin in diabetic rats were significantly changed in the presence of atorvastatin. The present study concludes that co-administration of atorvastatin with sitagliptin significantly improved the responses of sitagliptin in diabetic rats. Improved glucose metabolism and increased sitagliptin levels due to competitive inhibition of CYP $3 A 4$ enzyme by atorvastatin may be responsible for the improved anti-hyperglycemic activity of sitagliptin.
\end{abstract}

Keywords: Sitagliptin; atorvastatin; Type2 diabetes mellitus; DPP-4 inhibitor; HPLC; PK/PD parameters

\section{Introduction}

Diabetes mellitus is a complex metabolic disease affecting about $5 \%$ of people all over the world. According to the classification proposed by the American Diabetes Association, diabetes is divided into different types [1]. Type 1 diabetes accounts for $5-10 \%$ of all diabetic cases and results from the autoimmune destruction of the pancreatic $\beta$-cells. This type of diabetes usually develops rapidly because of the grave destruction of the insulin-secreting cells and patients are dependent on exogenous insulin. Type-II diabetes is more frequent, accounts for 90 $95 \%$ of all diabetic cases characterized by insulin resistance and relative insulin deficiency. Frequently accompanied by overweight or obesity. Hyperglycemia usually develops slowly and at earlier stages, blood glucose is moderately elevated. In Type-II diabetes, insulin resistance is initially compensated by increased secretion of insulin; however, this prolonged over stimulation of insulin secretion leads over time to progressive exhaustion and degradation of $\beta$-cells [2,3]. Dyslipidemia is common in patients with Type-II diabetes. Dyslipidemia is an abnormal amount of lipids (e.g. cholesterol and/or fat) in the blood.

Sitagliptin is an orally-active, potent and highly selective inhibitor of the Dipeptidyl peptidase 4 (DPP-4) enzyme, used in the treatment of Type-II diabetes. The DPP-4 inhibitors are class of agents that act as incretin enhancers. By inhibiting the DPP-4 enzyme, sitagliptin increases the levels of two known active incretin hormones, glucagonlike peptide-1 (GLP-1) and glucose-dependent insulinotropic polypeptide (GIP) [4]. Incretins are part of an endogenous system involved in the physiologic regulation of glucose homeostasis. When blood glucose concentrations are normal or elevated, GLP-1 and GIP increase insulin synthesis and release from pancreatic $\beta$ cells. GLP- 1 also lowers glucagon secretion from pancreatic $\alpha$ cells, leading to reduced hepatic glucose production. This mechanism is unlike the mechanism seen with sulfonylureas; sulfonylureas cause insulin release even when glucose levels are low, which can lead to sulfonylurea-induced hypoglycaemia in patients with type 2 diabetes and in normal subjects [5]. Sitagliptin is a potent, highly selective inhibitor of the enzyme DPP-4 and does not inhibit the closely-related enzymes DPP-8 or DPP9 at therapeutic concentrations [6,7]. Sitagliptin differs in chemical structure and pharmacological action from GLP-1 analogues, insulin, sulfonylureas or meglitinides, biguanides, peroxisome proliferatoractivated receptor gamma (PPAR $\gamma)$ agonists, alpha-glucosidase inhibitors and amylin analogues.

Dyslipidemia is common in patients with type 2 diabetes [8] and statins remain as the first choice of drugs in the treatment of diabetic dyslipidemia [9]. Atorvastatin represents a first line treatment option, alongside other hydroxyl methyl glutaryl coenzyme A (HMG$\mathrm{CoA})$ reductase, dose dependently lowers both cholesterol $[10,11]$ and triglyceride [12] levels in hyperlipidemic patients. Atorvastatin produces larger reductions of cholesterol and triglycerides compared with other drugs of the same class [13].

The study of mechanisms of drug interaction is of much value in diabetes mellitus therapy, because this is one of the metabolic disorders that needs treatment for prolonged periods and maintenance of normal

*Corresponding author: Venkateshwarlu Eggadi, Department of Pharmacology Hanamkonda, Warangal, Telangana, India. 506009. Tel: +919848835092: Fax +918702460108: E-Mail: eggadivenkey@gmail.com

Received December 17, 2014; Accepted December 31, 2014; Published January 07,2015

Citation: Eggadi V, Sheshagiri SBB, Devandla A, Dasi N, Kulundaivelu U, et al. (2015) Effect of Atorvastatin on Pharmacology of Sitagliptin in StreptozotocinNicotinamide Induced Type-II Diabetes in Rats. Biol Med (Aligarh) 6: 225. doi: 10.4172/0974-8369.1000225

Copyright: $\odot 2015$ Eggadi V, et al. This is an open-access article distributed unde the terms of the Creative Commons Attribution License, which permits unrestricted use, distribution, and reproduction in any medium, provided the original author and source are credited. 
blood glucose level is very important in this condition.Since both hyperglycemia and hypoglycemia are unwanted phenomena, there is every possibility for the combined use of sitagliptin and atorvastatin in diabetic dyslidemia [14]. Hence the present study was aimed at investigating the effect of atorvastatin on the activity of sitagliptin in diabetes induced rats. To evaluate the safety and effectiveness of the combination with respect to blood glucose levels.

\section{Materials and Methods}

Wistar rats of either sex of 8-9 weeks of age, weighing between 190 $\pm 20 \mathrm{~g}$ were used for the study. They were procured from Mahaveer agencies, Hyderabad, India. Animals were maintained under standard laboratory conditions at an ambient temperature of $25 \pm 2^{\circ} \mathrm{C}$ and 50 $\pm 15 \%$ relatively humidity with a $12 \mathrm{~h}$ light/dark cycle. Animals were fed with commercial standard pellet diet (Rayan's Biotechnologies Pvt Ltd., Hyderabad, India) and water ad libitum. Animals were fasted for $18 \mathrm{~h}$ prior to the experiment and during the experiment they were withdrawn from food and water. The animal experimental procedures and protocols were carried out according to the guidelines provided by the Committee for the Purpose of Control and Supervision of Experiments on Animals (CPCSEA).

\section{Study Design}

In clinical practice, both Sitagliptin and Atorvastatin were administered orally $[15,16]$. Hence, human oral therapeutic doses of the respective drugs were extrapolated to rat, based on body surface area [17]. The dose of Sitagliptin and Atorvastatin for rat experiments selected were $10.4 \& 20.0 \mathrm{mg} / \mathrm{kg}$ body weights respectively. Based on the dose-effect relationship of sitagliptin on blood glucose in normal rats. Sitagliptin and Atorvastatin were suspended in $0.5 \% \mathrm{CMC}$, for oral administration [18].

\section{Induction of diabetes in rats}

Experimental diabetes (NIDDM) was induced in overnight fasted adult Wistar strain albino rats weighing $190 \pm 20 \mathrm{~g}$ by a single i.p. injection of $60 \mathrm{mg} / \mathrm{kg}$ Streptozotocin (in citrate buffer, $\mathrm{pH} 4.5$ ), $15 \mathrm{~min}$ after the i.p. administration of $120 \mathrm{mg} / \mathrm{kg}$ of nicotinamide (in normal saline). Hyperglycemia was confirmed by the elevated glucose levels in plasma determined at $72 \mathrm{~h}$ and then on day 7 after injection. Plasma was collected by centrifuging the blood samples at a speed of 4000rpm for $15 \mathrm{~min}$. $10 \mu \mathrm{l}$ of plasma and $1 \mathrm{ml}$ of working reagent (GOD-POD) were mixed and incubated for $15 \mathrm{~min}$ at $37^{\circ} \mathrm{C}$. The absorbance of sample and standard (provided by manufacturer) was measured against blank at $505 \mathrm{~nm}$. Rats showing fasting plasma glucose levels $\geq 220 \mathrm{mg} / \mathrm{dl}$ (diabetic) were selected for study [19].

Diabetic albino Wistar rats were selected randomly and divided into 5 groups of 6 animals in each. Before the experiment, all animals were fasted for $18 \mathrm{hr}$ and water provided at ad libitum. Water was withdrawn during the experiment. After collection of initial blood samples, drugs were administered in the following order.

Group I: Diabetic control (Treated with $0.1 \mathrm{ml}$ of $0.5 \%$ of CMC) per 7days

Group II: Treated with sitagliptin (10.4 mg/kg; p.o.) per 7 days

Group III: Treated with atorvastatin $(20 \mathrm{mg} / \mathrm{kg}$; p.o.) per 7 days

Group IV: Treated with atorvastatin $(10.4 \mathrm{mg} / \mathrm{kg}$; p.o.) after $1 \mathrm{~h}$ followed by sitagliptin ( $20 \mathrm{mg} / \mathrm{kg}$; p.o.), (Single dose interaction studies; SDIs) per one day.
Group V: Pre-treated with atorvastatin $(20 \mathrm{mg} / \mathrm{kg}$; p.o.) for 7 days, on the $8^{\text {th }}$ day after $1 \mathrm{hr}$ administration of atorvastatin $(20 \mathrm{mg} / \mathrm{kg}$; p.o.), sitagliptin (10.4 mg/kg; p.o.) was given.

Blood samples $(1 \mathrm{ml})$ were collected by retro-orbital plexus at time intervals $0,1^{\text {st }}, 2^{\text {nd }}, 4^{\text {th }}, 8^{\text {th }}, 12^{\text {th }}$ and $24^{\text {th }}$ h using heparinized capillaries into a micro centrifugation tube containing anticoagulant (sodium citrate). Plasma was separated by centrifugation and stored at $-20^{\circ} \mathrm{C}$ until further analysis. These plasma samples were analyzed for blood glucose by GOD/POD method [20] and for plasma sitagliptin by HPLC method [21,22] (A gradient High Pressure Liquid Chromatography (Shimadzu Japan) equipped with C18 reversed-phase column). Hydrocortisone was used as internal standard and the mobile phase consist of methanol:water $(60: 40, \mathrm{v} / \mathrm{v}$, containing $10 \mathrm{mMTris}$ and 10 mMTriethylamine) was titrated to $\mathrm{pH} 9.0$ using $1 \mathrm{~mol} / \mathrm{L}$ hydrochloric acid. The mobile phase was eluted at a flow rate $1 \mathrm{ml} / \mathrm{min} \mathrm{C} 18$ reversedphased column at $30^{\circ} \mathrm{C}$ and the effluent was monitored at a wavelength of $267 \mathrm{~nm}$. The ratio of peak areas of Sitagliptin to that of internal standard was used for the quantification of Sitagliptin in plasma samples. The HPLC method was validated in terms of reproducibility, system suitability, recovery, accuracy, and precision and then applied for the estimation of sitagliptin in rat plasma. Maximum plasma concentration $\left(\mathrm{C}_{\max }\right)$ was the peak plasma concentration of a drug after administration, Time of peak plasma concentration $\left(\mathrm{T}_{\max }\right)$ was time to reach peak plasma concentration of a drug after administration, The area under the plasma drug concentration-time curve (AUC) reflects the actual body exposure to drug after administration of a dose of the drug, Half-life $\left(t_{1 / 2}\right)$ was the time required for the concentration of the drug to reach half of its original value, Mean residence time (MRT) was the time required for $63.2 \%$ of the dose to be eliminated from the body, Clearance $(\mathrm{Cl})$ was the volume of plasma cleared of the drug per unit time and Volume of distribution $\left(\mathrm{V}_{\mathrm{d}}\right)$ was theoretical volume in which the total amount of drug would need to be uniformly distributed to produce the desired blood concentration of a drug.

\section{Data and statistical analysis}

Data were expressed as mean \pm SD. The significance was determined by applying the Student's paired $t$ test. Significance values were found to be ${ }^{*} \mathrm{P}<0.05,{ }^{* *} \mathrm{P}<0.01,{ }^{* * *} \mathrm{P}<0.001$ compared to diabetic control. The plasma concentration-time data were analyzed by non-compartmental analysis using the Kinetica Software (version 5.0 from Thermo Fisher scientific Inc, USA).

\section{Results}

Sitagliptin produced antihyperglycemic activity with maximum percentage reduction $(42.75 \pm 1.24 \%)$ at $2 \mathrm{hr}$ in diabetic rats. The average percentage blood glucose reduction with sitagliptin, atorvastatin and their combination in diabetic rats is shown in Table 1. Atorvastatin alone has no significant effect on the blood glucose level of diabetic rats (Table 1). When administered in combination with sitagliptin, atorvastatin significantly increased the effect of sitagliptin in diabetic rats $(42.75$ vs $51.80 ; P<0.01)$. The average plasma sitagliptin concentrations alone and in combination with atorvastatin in diabetic rats were shown in (Table 2). The mean PK parameters of sitagliptin alone and in combination with atorvastatin in diabetic rats are shown in Table 3. The plasma sitagliptin levels at 1, 2, 4, 8, 12, and 24hrs intervals were increased in the presence of atorvastatin and the PK parameters, such as $\mathrm{C}_{\max }, \mathrm{T}_{\max }, \mathrm{t}_{1 / 2}, \mathrm{Cl}, \mathrm{V}_{\mathrm{d}}, \mathrm{AUC}$ and MRT were altered significantly in the presence of atorvastatin in diabetic rats. 
Citation: Eggadi V, Sheshagiri SBB, Devandla A, Dasi N, Kulundaivelu U, et al. (2015) Effect of Atorvastatin on Pharmacology of Sitagliptin in Streptozotocin- Nicotinamide Induced Type-II Diabetes in Rats. Biol Med (Aligarh) 6: 225. doi: 10.4172/0974-8369.1000225

Page 3 of 4

\begin{tabular}{|c|c|c|c|c|c|}
\hline Time & Diabetic control & Sitagliptin & Atorvastatin & Sitagliptin + Atorvastatin (SDI) & Sitagliptin + Atorvastatin (MDI) \\
\hline 0 & 00.00 & 00.00 & 00.00 & 00.00 & 00.00 \\
\hline 1 & $0.92 \pm 0.04$ & $27.28 \pm 0.85^{*}$ & $12.15 \pm 0.35$ & $31.38 \pm 1.50^{*}$ & $37.62 \pm 1.47^{*}$ \\
\hline 2 & $1.56 \pm 0.12$ & $42.75 \pm 1.24^{* *}$ & $21.69 \pm 0.89$ & $45.94 \pm 1.20^{*}$ & $51.80 \pm 2.58^{* *}$ \\
\hline 4 & $1.66 \pm 0.20$ & $49.57 \pm 1.98^{* *}$ & $27.24 \pm 1.02$ & $64.46 \pm 3.47^{*}$ & $64.10 \pm 2.98^{* *}$ \\
\hline 8 & $2.03 \pm 0.35$ & $35.45 \pm 1.24^{*}$ & $17.35 \pm 0.25$ & $47.12 \pm 2.51^{*}$ & $41.49 \pm 1.87^{*}$ \\
\hline 12 & $2.29 \pm 0.42$ & $24.29 \pm 1.5^{*}$ & $8.99 \pm 0.27$ & $42.32 \pm 2.05^{*}$ & $29.85 \pm 0.98^{*}$ \\
\hline 24 & $2.46 \pm 0.35$ & $17.82 \pm 1.68^{*}$ & $4.54 \pm 0.05$ & $26.94 \pm 0.98^{*}$ & $34.40 \pm 0.41^{*}$ \\
\hline
\end{tabular}

Data were expressed as mean \pm SD. The significance was determined by applying the Student's paired test. Significance values were found to be ${ }^{*} P<0.05$, " $P<0.01$, ${ }^{* *+} P<0.001$ compared to diabetic control. (SDI-Single Dose Interaction, MDI-Multi Dose Interaction).

Table 1: Percentage of glucose reduction in diabetic rats after oral administration of Sitagliptin, Atorvastatin alone and in combination (SDI \& MDI).

\begin{tabular}{|c|c|c|c|}
\hline Time (hr) & Sitagliptin & Sitagliptin+Atorvastatin (SDI) & Sitagliptin+Atorvastatin (MDI) \\
\hline 0 & 00.00 & 00.00 & 00.00 \\
\hline 1 & $3.38 \pm 0.39$ & $5.02 \pm 0.81^{* *}$ & $12.31 \pm 0.96^{* *}$ \\
\hline 2 & $7.01 \pm 0.42$ & $5.98 \pm 0.46^{* *}$ & $9.49 \pm 0.85^{*}$ \\
\hline 4 & $3.92 \pm 0.26$ & $2.61 \pm 0.51^{* *}$ & $4.68 \pm 0.69^{*}$ \\
\hline 8 & $1.19 \pm 0.25$ & $0.92 \pm 0.24$ & $2.54 \pm 0.72^{*}$ \\
\hline 12 & $0.77 \pm 0.18$ & 00.00 & $1.05 \pm 0.41$ \\
\hline
\end{tabular}

Data were expressed as mean \pm SD and analyzed statistically by using one way ANOVA followed by Dunnett's test. Significance values were found to be ${ }^{*}<<0.05$ ${ }^{* *} P<0.01,{ }^{* *} P<0.001$ compared to diabetic control. (SDI-Single Dose Interaction, MDI-Multi Dose Interaction).

Table 2: Concentration of sitagliptin in rat plasma at different time intervals in sitagliptin and combination groups (SDI, MDI) using Kinetica 5.0.

\begin{tabular}{|c|c|c|c|}
\hline PK parameters & Sitagliptin & Sitagliptin+Atorvastatin (SDI)(Day1) & Sitagliptin+Atorvastatin (MDI) (Day8) \\
\hline Cmax & $6.74 \pm 0.42$ & $11.07 \pm 0.79^{* *}$ & $2 \pm 0$ \\
\hline$T_{\max }$ & $2 \pm 0$ & $54.95 \pm 2.31^{* *}$ & $5.89 \pm 1.41^{* *}$ \\
\hline AUC & $33.58 \pm 2.49$ & $2.96 \pm 0.43$ & $3.97 \pm 1.23$ \\
\hline $\mathbf{t}_{1 / 2}$ & $3.19 \pm 0.45$ & $5.03 \pm 0.53$ & $6.2 \pm 1.48$ \\
\hline MRT & $5.17 \pm 0.51$ & $142.90 \pm 7.43^{* *}$ & $160.88 \pm 21.69^{* *}$ \\
\hline CI & $233.96 \pm 14.43$ & $610.87 \pm 75.98^{* *}$ \\
\hline $\mathbf{V}_{d}$ & $1072.53 \pm 93.78$ & $2524.02 \pm 109.82^{* *}$ \\
\hline
\end{tabular}

Data were expressed as mean \pm SD and analyzed statistically by using one way ANOVA followed by Dunnett's test. Significance values were found to be ${ }^{*}<0.05$, ${ }^{* *} P<0.01,{ }^{* *} P<0.001$ compared to diabetic control. (SDI-Single Dose Interaction, MDI-Multi Dose Interaction). Cmax-Maximum drug concentration, $T_{\text {max }}{ }^{-T i m e ~ o f ~ p e a k ~ p l a s m a ~}$ concentration, AUC- area under the curve, MRT- Mean Residence Time, Cl- Clearance, $\mathrm{V}_{\mathrm{d}}$-Volume of Distribution.

Table 3: Various pharmacokinetic parameters (Mean\& SD) of sitagliptin alone and in combination with atorvastatin (SDI \& MDI) in diabetic rats at different time intervals (using Kinetica 5.0.)

\section{Discussion}

Drug interactions are usually seen in clinical practice and mechanisms of interactions are evaluated usually in animal models (rodents and no-rodents). The statin derivatives were studied in lipidlowering action and glucose metabolism effect in hyper-cholesterolemic patients with concurrent diabetes [23] and also canagliflozin versus sitagliptin for type $2 \mathrm{DM}$ [24]. In the present study, influence of atorvastatin on the activity of sitagliptin in diabetic rats was studied. Since small amount of plasma was required for glucose analysis, the blood samples were collected by retro-orbital puncture [25].The present study revealed no or very little glucose-lowering effect of atorvastatin alone in diabetic condition, with respect to pre dose glucose levels. The $\mathrm{PD}$ of sitagliptin was increased significantly $(\mathrm{P}<0.01)$ in the presence of atorvastatin in diabetic rats. The $\mathrm{PK}$ parameters $\left(\mathrm{C}_{\max }, \mathrm{T}_{\max }, \mathrm{T}_{1 / 2}, \mathrm{Cl}, \mathrm{V}_{\mathrm{d}}\right.$, AUC and MRT) of sitagliptin significantly changed in the presence of atorvastatin. This confirms synergistic interaction between atorvastatin and sitagliptin in diabetic rats. The impact of atorvastatin on the activity of sitagliptin was significant following single oral dose administration in diabetic rats. After oral administration the peak anti-hyperglycemic activity was observed at $2 \mathrm{~h}$ in diabetic rats. Sitagliptin is rapidly absorbed and has a half-life of approximately $8-14 \mathrm{~h}$. Sitagliptin is extensively metabolized by the hepatic cytochrome P450 enzyme system (CYP3A4 and CYP2C8) with less than $2 \%$ of an oral dose being excreted unchanged in humans [25-27]. Atorvastatin is mainly metabolized by CYP3A4 and meanwhile atorvastatin also having the inhibitory effect against CYP3A4 mediated metabolism. The above literature supports, the presence of atorvastatin significantly increased the PK of sitagliptin in diabetic rats. These results showed that atorvastatin is an effective inhibitor of P-glycoprotein and CYP3A4 mediated metabolism in intestine and/or liver. Therefore, atorvastatin could affect the sitagliptin activity in diabetic rats by increasing plasma levels of the sitagliptin. Overall the interaction of sitagliptin and atorvastatin was mainly due $\mathrm{PK}$ in nature and increased blood glucose lowering effect of sitagliptin.

\section{Conclusion}

The present study concludes that co-administration of atorvastatin with sitagliptin significantly improves anti-hyperglycemic activity of sitagliptin when compared to sitagliptin alone treated StreptozotocinNicotinamide induced diabetic rats.

\section{References}

1. American Diabetes Association (2006) Diagnosis and classification of diabetes mellitus. Diabetic Care 29: 43-48.

2. American Diabetes Association (2002) Management of dyslipidemia in adults with diabetes. Diabetes Care 25: S74-S77.

3. Anil D, Rizwanbasha K, Jayasankar K, Venkat M, Malay K, et al. (2012) Bioanalytical method development and validation of sitagliptin phosphate by 
Citation: Eggadi V, Sheshagiri SBB, Devandla A, Dasi N, Kulundaivelu U, et al. (2015) Effect of Atorvastatin on Pharmacology of Sitagliptin in Streptozotocin- Nicotinamide Induced Type-II Diabetes in Rats. Biol Med (Aligarh) 6: 225. doi: 10.4172/0974-8369.1000225

Page 4 of 4

RP-HPLC and its application to pharmacokinetic study. Inter J Pharm Pharm Sci 4: 18-22.

4. Anne Kelly, Spratt DO (2009) Managing diabetic dyslipidemia: aggressive approach. J Amer Osteopath Ass 109: S2-S7.

5. Bakker-Arkema RG, Davidson MH, Goldstein RJ, Davignon J, Isaacsohn $\mathrm{JL}$, et al. (1996) Efficacy and safety of a new HMG-CoA reductase inhibitor, atorvastatin, in patients with hypertriglyceridemia. JAMA 275: 128-133.

6. Chwieduk CM (2011) Sitagliptin/metformin fixed-dose combination: in patients with type 2 diabetes mellitus. Drugs 71: 349-361.

7. Connor M, Kitchen I (2006) Has the sun set on kappa3-opioid receptors? Br J Pharmacol 147: 349-350.

8. Dhillon S (2010) Sitagliptin: a review of its use in the management of type 2 diabetes mellitus. Drugs 70: 489-512.

9. Eckel RH, Wassef M, Chait A, Sobel B, Barrett E, et al. (2002) Prevention Conference VI: Diabetes and Cardiovascular Disease: Writing Group II: pathogenesis of atherosclerosis in diabetes. Circulation 105: e138-143.

10. Hansen JB, Arkhammar PO, Bodvarsdottir TB, Wahl P (2004) Inhibition of insulin secretion as a new drug target in the treatment of metabolic disorders. Curr Med Chem 11: 1595-1615.

11. Jones P, Kafonek S, Laurora I, Hunninghake D (1998) Comparative dose efficacy study of atorvastatin versus simvastatin, pravastatin, lovastatin, and fluvastatin in patients with hypercholesterolemia (the CURVES study) Am J Cardiol 81: 582-587.

12. Lau YY, Okochi H, Huang Y, Benet LZ (2006) Pharmacokinetics of atorvastatin and its hydroxy metabolites in rats and the effects of concomitant rifampicin single doses: relevance of first-pass effect from hepatic uptake transporters, and intestinal and hepatic metabolism. Drug Metab Dispos 34: 1175-1181.

13. Lencioni C, Lupi R, Del Prato S (2008) Beta-cell failure in type 2 diabetes mellitus. Curr Diab Rep 8: 179-184.

14. Lyseng-Williamson KA (2007) Sitagliptin. Drugs 67: 587-597.

15. Maria G, Christopher J, David Q, James R (2006) Disposition of the Dipeptidy Peptidase 4 Inhibitor Sitagliptin in Rats and Dogs. The Americ Soc Pharmaco Exp Therap 35: 525-532.
16. Nawrocki JW, Weiss SR, Davidson MH (1995) Reduction of LDL cholestero by $25-60 \%$ in patients with primary hypercholesterolemia by atorvastatin, a new HMG-CoA reductase inhibitor. Arterioscler Thromb Vasc Biol 15: 678-682.

17. Nielsen KK, Bjornsdottir I, Andersen JV, Thomsen MS, Hansen KT (2001) Pharmacokinetics and metabolism of $14 \mathrm{C}$-sitagliptin after a single oral dose to healthy Japanese and Caucasian males. ArteriosclerThrombVascBiol69: 88

18. Paget GE, Barnes JM (1964) Toxicity tests. In: Lawrence DR, Bacharach AL (Eds) Evaluation of drug activities: pharmacometrics. Academic Press, London 140-161.

19. Phaneemdra D, Venkatesh V, Ramarao N (2012). Simultaneous estimation of simvastatin and sitagliptin by using different analytical methods. Inter J Adv in Pharm Anal 2: 19-23.

20. RILEY $\vee(1960)$ Adaptation of orbital bleeding technic to rapid serial blood studies. Proc Soc Exp Biol Med 104: 751-754.

21. Satyanarayana S1, Kilari EK (2006) Influence of nicorandil on the pharmacodynamics and pharmacokinetics of gliclazide in rats and rabbits. Mo Cell Biochem 291: 101-105.

22. Scheen AJ1 (2010) Pharmacokinetic and pharmacodynamic evaluation of sitagliptin plus metformin. Expert Opin Drug MetabToxicol 6: 1265-1276.

23. Ektare VU, Lopez JM, Martin SC, Patel DA, Rupnow MF, et al. (2014) Cost efficiency of canagliflozin versus sitagliptin for type 2 diabetes mellitus. Am J Manag Care 20: s204-215.

24. Ogawa H1, Matsui K, Saito Y, Sugiyama S, Jinnouchi H, et al. (2014) Differences between rosuvastatin and atorvastatin in lipid-lowering action and effect on glucose metabolism in Japanese hypercholesterolemic patients with concurrent diabetes. Lipid-lowering with highly potent statins in hyperlipidemia with type 2 diabetes patients (LISTEN) study â€“. Circ J 78: 2512-2515.

25. Szkudelski T1 (2012) Streptozotocin-nicotinamide-induced diabetes in the rat Characteristics of the experimental model. ExpBiol Med (Maywood) 237: 481 490.

26. Trinder $P$ (1969) Determination of blood glucose using an oxidase-peroxidase system with a non-carcinogenic chromogen. J ClinPathol 22: 158-161.

27. Wilde Michelle I, Spencer Caroline M (1998) Management of dyslipidemias the potential role of atorvastatin. Disease Manage Health Outcomes 3: 293-311. 